\title{
Immuno-radiotherapy for brain metastases
}

\author{
Denaro Nerina $^{1^{*}}$, Occelli marcella ${ }^{1}$, Merlotti anna maria ${ }^{2}$, Cauchi Carolina ${ }^{1}$ and Merlano Marco Carlo $^{1}$ \\ ${ }^{1}$ Oncology Department, S. Croce \& Carle Teaching Hospital Cuneo, Italy \\ ${ }^{2}$ Radiation Oncology Department, S. Croce \& Carle Teaching Hospital Cuneo, Italy
}

\begin{abstract}
Brain metastases $(\mathrm{BM})$ are a lethal consequence of systemic dissemination of cancer. The most common malignancies that metastasize to the brain are lung, breast, and melanoma. Eighty percent of patients present with brain metastases within the cerebral hemispheres; $15 \%$ are within the cerebellar hemispheres and $5 \%$ are within the brainstem. The incidence of brain metastases is increasing because of better detection from improved imaging techniques (also for staging asymptomatic patients), and more effective systemic treatment regimens that can prolong life, permitting the cancer to disseminate to the brain, a sanctuary site.
\end{abstract}

\section{Introduction}

Prognostic scores as an estimation of a patient's prognosis may guide therapy customized for these patients. Different outcomes have been shown in multiple studies in patients stratified with different prognostic scoring systems: most of them have some limitations considering primarily age, KPs and number of BM in multiple primary subsytes. The role of genetic signature and other tumor-related features in BM remains unclear [1-4].

One of the challenges in the development of effective therapies for $\mathrm{BM}$ is the presence of the blood-brain barrier(BBB), a highly selective permeability barrier made of capillary endothelial cells connected by tight junctions and astrocyte foot processes that limit entry of systemic therapies into the brain [5]. In addition, active transport mechanisms of drug efflux and high plasma protein binding of agents further lower the volume of distribution of agents in the brain parenchyma [6].

However BBB is not efficient in metastatic disease and several studies showed that BBB permeability changed over time as BM developed [7].

Due to the long cancer history patients with BM are usually heavily pretreated with tumors that are more likely to be resistant to therapy. Median overall survival (OS) from diagnosis of BM ranges from 3 to 6 months [8].

The standard therapeutic options include surgery, whole brain radiation therapy (WBRT), stereotactic radiation (SRS), systemic therapy-and symptom management only.

The role of systemic therapy (chemotherapy, targeted agents, or immunotherapy) in brain metastases is palliation, because there is no level-1 evidence favouring the use of systemic therapy compared with local approaches, systemic therapies are used when disease control rate is researched also in other sites. In recent years, the development of novel cytotoxic agents and targeted therapies with better blood-brain barrier penetration have increased the interest in use of systemic therapies in brain metastases. Although patients with BM are under represented or excluded from the majority of clinical trials testing new drugs. Considering the tumours with most common cerebral involvement: $10-40 \%$ of patients with metastatic melanoma develop BM during their lifetime and $>75 \%$ have $\mathrm{BM}$ at autopsy, with median survival in the order of 2.5-4 months despite use of WBRT and surgery. Among those with NSCLC, $10 \%$ have brain metastases at presentation and another $30 \%$ develop them over the course of their disease. Survival after the development of brain metastases is as dismal in those with NSCLC as it is for melanoma. Multifocal disease is common in both of these diseases, with about half of patients with CNS disease presenting with more than one brain lesion [9].

Advances in the understanding of the biology of BM and molecularly defined disease subsets have facilitated an emerging role of novel therapeutic agents, such as targeted therapies and immunotherapy. In particular, there is a great interest in the association between radiation treatment and immunotherapy especially given case reports and animal studies that suggest combined treatment may generate abscopal responses outside the radiation field.

A limit in the development of immunotherapy is the large use of steroids in this settings; steroids (dexamethasone) are recommended in symptomatic BM to provide temporary symptomatic relief of symptoms related to increased intracranial pressure and edema. Steroids have a wide immunosuppressive action, including reduction of CD8+,CD4+cells and monocyte/neutrophils ratio (depending on the expression of GCR), macrophage dysfunction and $\mathrm{T}_{\text {reg }}$ induction [10]. Although successful clinical experience would lead to the conclusion that the role of steroids is firmly established in the management of brain metastases, there is a great variability in recommendations and a general lack of well-controlled studies addressing this specific issue. Moreover no clear evidence supported a contraindication of combining steroids with immunotherapy.

The aim of this review is to point out the main experiences on treatment of $\mathrm{BM}$ with radiation and the possible development of immune radiotherapy combination.

Correspondence to: Denaro Nerina, Oncology Department, S. Croce \& Carle Teaching Hospital Cuneo, Italy, Tel: +390171616350; Fax: +390171616360; E-mail: nerinadenaro@gmail.com

Key words: brain metastases, ipilimumab, pembrolizumab, avelumab, atezolizumab, nivolumab, radiotherapy, radiosurgery, immunotherapy

Received: April 26, 2017; Accepted: May 15, 2017; Published: May 18, 2017 


\section{Methods}

Preferred Reporting Items for Systematic Reviews and MetaAnalyses (PRISMA) guidelines were used for the conduct and reporting of this systematic review [11].

\section{Search strategy}

Studies were identified by a computerized search of the PubMed data-base with the string "brain metastases", radiotherapy AND "(ipilimumab OR pembrolizumab OR avelumab OR atezolizumab OR nivolumab)" (Figure 1). The search was performed on December 2016. Moreover, abstracts published by the ASCO,ESMO and ESTRO between 2010 and 2016 were reviewed and considered for inclusion if full paper was not published. Reference lists of original articles and review articles were considered as additional sources of information (Figure 1).

\section{Inclusion and exclusion criteria}

English-language studies which reported any survival outcome (median OS and/or landmark analysis of survival) of patients treated with immunotherapy and BM were included in this review. The following information was extracted from each report: tumor primary, number of patients with $\mathrm{BM}$, treatment regimens and line of treatment (i.e. pre-treated or treatment-naïve patients), previous BM local treatment, median OS, landmark analysis of survival, median progression-free survival (PFS) in brain?, overall response rate, disease control rate, intracranial response rate, presence of "central nervous system" (CNS) symptoms, incidence of G3-4 toxicities, discontinuation rate, CNS specific adverse events (for safety outcomes, we included studies for patients with BM only or reporting outcomes for BM patients separately).

Exclusion criteria for study selection were the following: (1) studies investigating the immuno checkpoint not involving brain metastases or radiotherapy; (2) those published in language other than English; (3) duplicate publications.

\section{Extraction and analysis}

Data were independently extracted by 4 investigators (N.D., MO, $\mathrm{CC}$ and AM.). To ensure homogeneity of collection and to rule out the effect of subjectivity in data gathering and entry, disagreements were resolved by interaction, discussion, and consensus. Analysis of extracted data was performed by all the Authors. Revision was performed by MM.

\section{Biological background}

Immune checkpoint targeting used sequentially with RT in melanoma/lung brain metastases may potentiate durable brain response [12]. The optimal treatment sequence has not been delineated. Several pre-clinical studies have demonstrated the variable permeability of the BBB to immune cells, in pathological settings such as septic encephalopathy, experimental autoimmune encephalomyelitis (EAE), or tumorigenesis [13]. Moreover a lymphatic communication exists between the CNS and the periphery (from the brain parenchyma, along the Virchow-Robbins spaces, through the cribriform plate, and out to the deep cervical lymph nodes). $[14,15]$. Subsequent studies found that APCs (antigen presenting cells) could migrate from the brain to the cervical lymph nodes, and that the type and extent of inflammation in the brain correlates with the frequency of CNS antigens found in those draining nodes [16].

Furthermore, while in brain parenchyma immune population seems to be not significant, innate and adaptive immune reactivity in the ventricles, leptomeninges, and perivascular spaces is similar to that seen in the periphery [17].

In brain disease (both tumoral and non tumoral) effector $\mathrm{T}$ cells are capable of entering the parenchyma to proliferate, home or migrate to the tumor, and initiate an active inflammatory response or pro versus antitumoral treatment. Goldberg et al evidenced that T-cells are an integral part of the CNS and shape its function via the choroid plexus.

This strong biologic background led in the last few years to a new interest on immunotherapy also in brain cancers (primitive and secondary). Although only a few of the growing number of checkpoint inhibitors (namely, CTLA-4 and PD-1) have been studied until now in this setting, the use of immune modulators appears promising.

A potential limit to immune therapy is the wide use of steroids in BM. The steroids are used specifically to decrease oedema associated with primary and secondary tumors of the central nervous system. The glucocorticoids (GCs) induce immunosuppressive and anti-inflammatory effects predominantly through binding to the

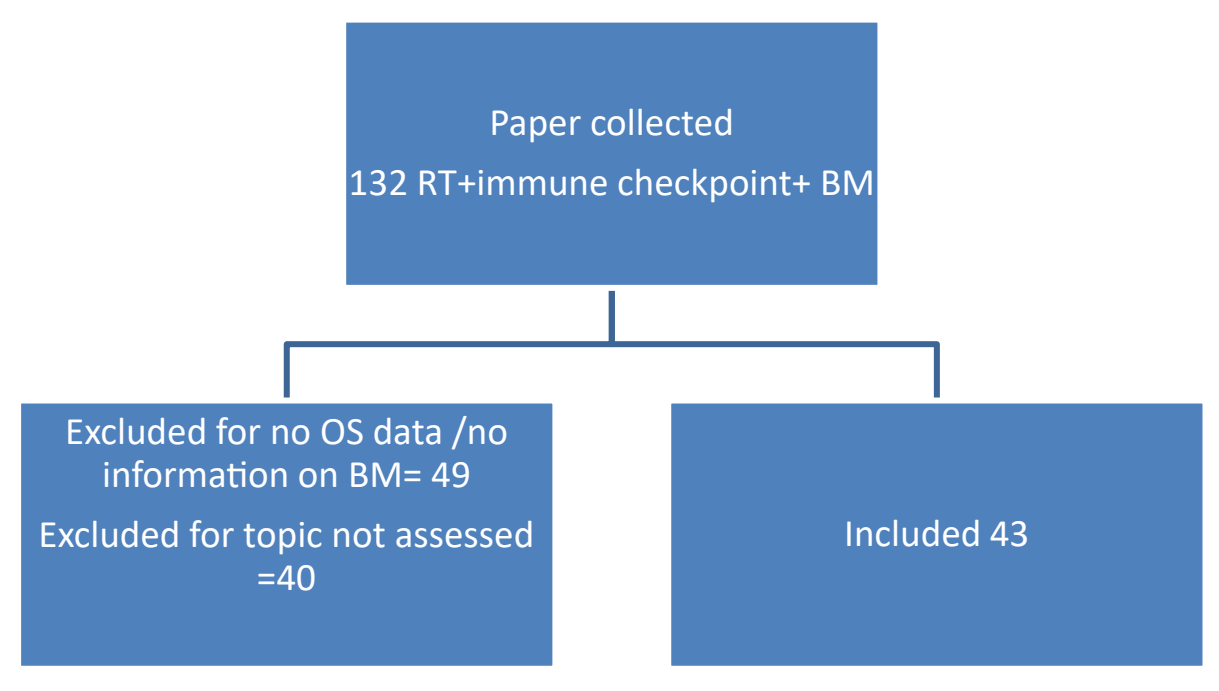

Figure 1. Inclusion and exclusion criteria. 
glucocorticoid receptor (GCR) [18]. It is known that dexamethasone and other GCs can modulate the immune system in a wide variety of ways, with varying mechanisms of action in different cell types $[19,20]$.

Dex -induced immunological effects, including a large-scale lymphodepletive effect particularly affecting CD4+ T cells but also $\mathrm{CD} 8+\mathrm{T}$ cells. The proportion of regulatory $\mathrm{T}$ cells within the $\mathrm{CD} 4 \mathrm{C}$ compartment did not change after Dex was administered, however a significant increase in proliferation and activation of regulatory $\mathrm{T}$ cells as well as a proportional changes in dendritic cell (DC) subtypes was observed.

However, no clinical data exist giving a clear evaluation of the immunosuppressive effect of steroids during immune therapy.

\section{Radiotherapy}

Radiotherapy uses high energy radiation to locally treat cancer inducing DNA damage and endoplasmic reticulum (ER) stress via reactive oxygen species (ROS) thus causing cycle arrest of the tumor cells that try to repair the induced damage and reducing their clonogenic potential. In addition to these targeted effects, an increased expression of MHC-I and MHC-II molecules, CD80, CD86 adhesion molecules, stress ligands, Hsp70 and death receptors on tumor cell surfaces can be observed. All these non-targeted effects may result in activation of death cascade and DCs activation [21].

The induction of immunogenic cancer cell death seems to be a common mechanism for most RT schemes. Activation of DCs is a process that has to be tightly controlled to avoid tolerance. In addition to lack of activation of DCs, toler4ance is also supported by a number of other mechanisms such as the expression of immune check-points. Cytotoxic T lympho- cyte antigen 4 (CTLA-4) and programmed cell death protein 1 (PD-1) on $\mathrm{T}$ cells are two major checkpoints.

\section{Immunotherapy}

Significant advances in treatment of metastatic solid tumors have been made with immunotherapy. Checkpoint inhibitors treatments represent a standard treatment in first and second line of melanoma and lung cancer and in second line of renal cancer.

Blockade of CTLA-4 (ipilumimab and tremelimumab), PD-1 (nivolumab, pembrolizumab, pidilizumab and others), and PDL1 (BMS 936559, durvalimumab Avelumab, and atezolizumab) can produce significant improvement in overall survival in several solid tumors. A better characterization of primary tumors and $\mathrm{BM}$ is necessary as mutational status of EGFR, levels of STAT3 and chemokines correlates with immunotherapy response.

For example in NSCLC patients with ALK-rearranged or EGFRmutated tumour exhibit low response to antibodies against PD-1 or PD-L1 compared with high responses in patients without known actionable mutations, probably because of the absence of CD8 T-cells [22].

\section{Ipilimumab}

Ipilimumab is a monoclonal antibody that antagonizes cytotoxic T-lymphocyte antigen-4 (CTLA-4), It has been optimum timing and sequencing of immunomodulation and radiation therapy in humans is undefined. Because the effects of ipilimumab and other types of immunotherapy can be both delayed in onset and prolonged over many years [23]. a warming immune response could potentially be boosted by radiation therapy even some time after the drug is discontinued. Timing was investigated in a retrospective study by Saraceni et al.
They reported a cohort of 30 patients in which patients who received immunotherapy $>30 \mathrm{~d}$ after RT had statistically significant better OS (median OS 58.00 vs. $34.72 \mathrm{wk}$ ), $\mathrm{p}=0.0493$. The majority of patients received anti-CTLA4 (76.67\%) and gammaknife (66.67\%). Brain responses included partial, $42.86 \%$; progressive, $35.71 \%$; stable, $14.29 \%$; and complete, $7.14 \%$ [24].

Radiation given prior to ipilimumab could ostensibly liberate antigen and recruit $\mathrm{T}$-cells to the tumor microenvironment as a priming event, which would later be amplified by checkpoint blockade. In the case of ipilimumab delivered first, radiotherapy could boost immunogenic cell death, as the host would have tumor-reactive T cells activated by initial treatment with checkpoint blockade. Further studies will be needed to understand and exploit such mechanisms. Results on combination with RT are summarized in Table 1.

\section{Pembrolizumab}

The anti-PD1 antibody pembrolizumab prolonged progressionfree survival and overall survival in advanced melanoma and in PDL1pos NSCLC and other advanced solid cancer (phase III studies on going).

In the registrative study KEYNOTE 006 8.2\%, 9.7\%,10.1\% had no-active brain metastases respectively in the biweekly and triweekly pembrolizumab arms and ipilimumab arm. However no data are separately reported about them. Recently results of a phase II trial NCT02085070 (pembrolizumab in patients with untreated brain metastases from melanoma or NSCLC) were published. Goldberg SB et al reported $22 \%$ brain metastasis response (95\% CI 7-48) in patients with melanoma and 33\%(95\% CI 14-59) in patients with NSCLC. Responses were durable, with all but one patient with NSCLC who responded showing an ongoing response at the time of data analysis on June 30, 2015 [22].

\section{Avelumab}

Avelumab is is a fully human IgG1 MAb targeting the co-regulatory protein PD-L1. Avelumab is shown to mediate antibody-dependent cell-mediated cytotoxicity (ADCC) of several types of human tumor cell lines (e.g., breast, lung, bladder carcinomas) in vitro, with tumor cell lysis mediated mainly by human CD16+ monocytes and natural killer (NK) cells [25]. Due to results of Phase I/II studies in patients with advanced cancer Avelumab is under investigation in several cancers such as Hodgkin, Merkel, Ovarian,Renal, Gastric, NSCLC,breast and urothelial cancer. No data are published on combination with RT in $\mathrm{BM}$ patients. (clinicaltrials.gov) http://www.ncbi.nlm.nih.gov/pmc/ articles/PMC4910121/ - bib16

\section{Atezolizumab}

Atezolizumab is an engineered humanized monoclonal immunoglobulin G1 antibody that binds selectively to PD-L1, and prevents its interaction with PD-1 and B7-1 (also known as CD80).

Efficacy and safety of atezolizumab has been demostrated in phase 2 trials in urothelial carcinoma, NSCLC, renal carcinoma, and other solid tumours. Phase 3 trials are ongoing in several diseases. The studies excluded patients who had symtomatic or corticosteroid-dependent brain metastases. And specifically, no outcome information about patients enrolled in the studies who had brain metastases or patient who had radiation therapy. In the phase 1a RCC expansion study (PCD4989g - RCC) included 70 patients with metastatic clear cell or non-clear cell renal carcinoma. In the baseline patient's characteristic 
Table 1. Patients treated with immunoradiotherapy in clinical trials

\begin{tabular}{|c|c|c|c|c|c|c|c|c|c|c|c|c|c|c|c|c|c|c|c|c|}
\hline $\begin{array}{c}\text { Study } \\
\text { (author, } \\
\text { year) }\end{array}$ & 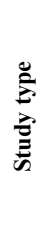 & 跑 & $\begin{array}{l}n \\
0 \\
0 \\
0 \\
z\end{array}$ & 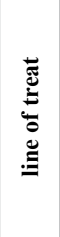 & 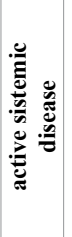 & 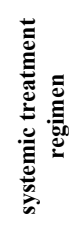 & 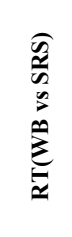 & 苞 & 总 & 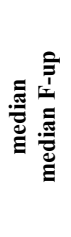 & 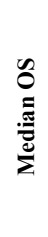 & 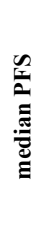 & ֶㅡㅁ & $\stackrel{\cong}{\cong}$ & $\begin{array}{l}0 \\
\dot{j} \\
0 \\
0 \\
0 \\
0 \\
\pm \\
0\end{array}$ & 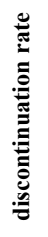 & 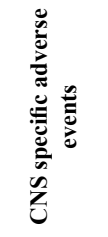 & 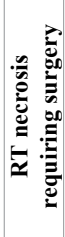 & 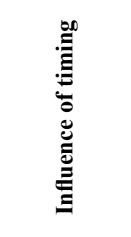 & की \\
\hline $\begin{array}{l}\text { Schoenfeld } \\
\text { et al. } 2015\end{array}$ & $\simeq$ & $\Sigma$ & $\stackrel{0}{\circ}$ & $\ddot{Z}$ & 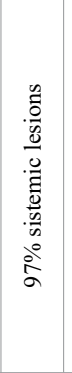 & 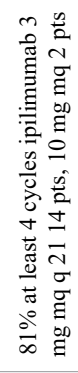 & 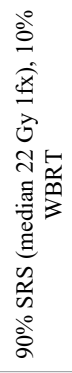 & 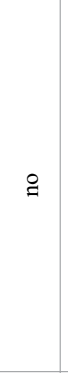 & 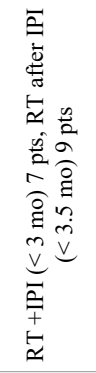 & $\vec{Z}$ & $\begin{array}{l}\stackrel{\bigcirc}{\Xi} \\
\stackrel{ \pm}{ \pm}\end{array}$ & $\Xi$ & $\breve{g}$ & $\bar{Z}$ & $\vec{\exists}$ & 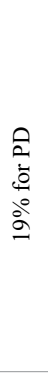 & $\vec{Z}$ & 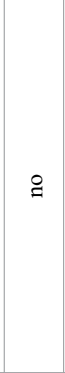 & 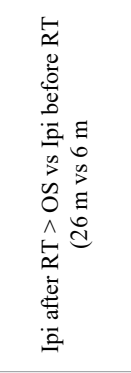 & 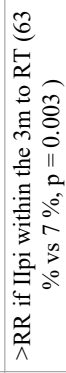 \\
\hline $\begin{array}{l}\text { Chandra } \\
2015\end{array}$ & $\simeq$ & $\Sigma$ & f & $\bar{Z}$ & $\Xi$ & 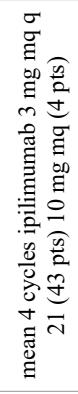 & 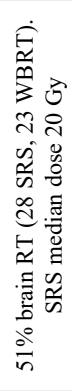 & 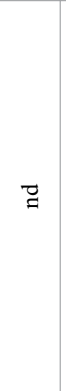 & 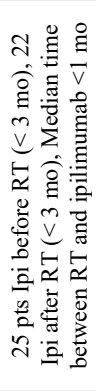 & 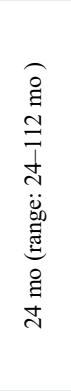 & 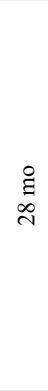 & $\bar{Z}$ & 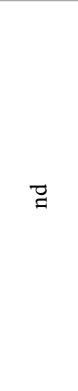 & $\bar{Z}$ & $\bar{Z}$ & $\bar{Z}$ & $\vec{z}$ & $\Xi$ & 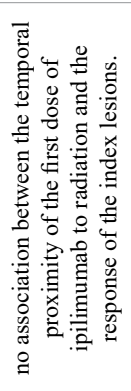 & 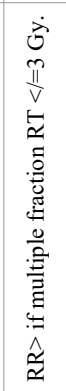 \\
\hline Kiess 2015 & $\simeq$ & $\Sigma$ & f & 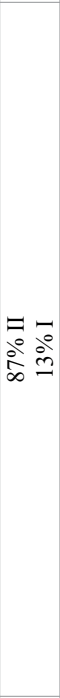 & $\vec{z}$ & 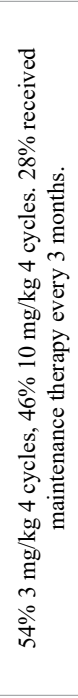 & 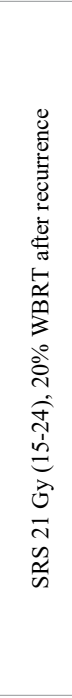 & 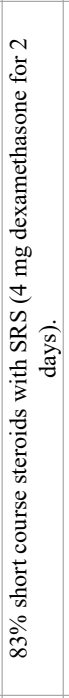 & 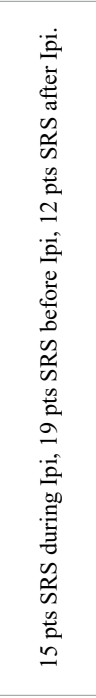 & 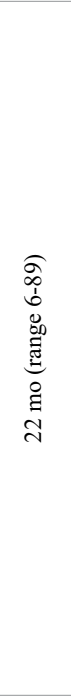 & 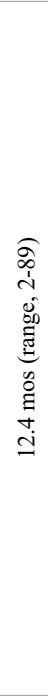 & $\Xi$ & 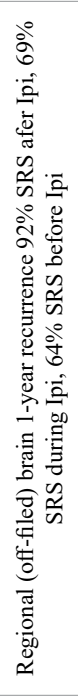 & $\vec{Z}$ & 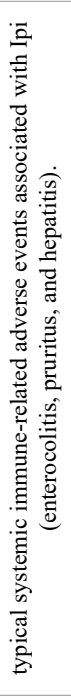 & $\Xi$ & 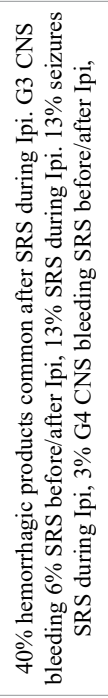 & $\begin{array}{l}\frac{n}{2} \\
\text { in }\end{array}$ & 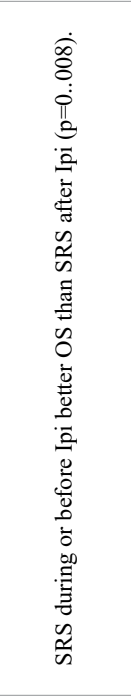 & $\vec{I}$ \\
\hline $\begin{array}{l}\text { Knisely } \\
2012\end{array}$ & $\simeq$ & $\Sigma$ & $\widehat{\sim}$ & $\begin{array}{l}\Xi \\
\vdots \\
\Xi \\
=\end{array}$ & $\Xi$ & 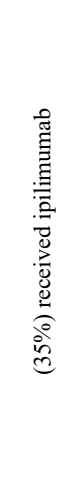 & 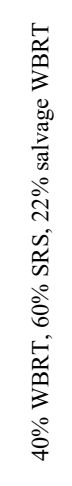 & 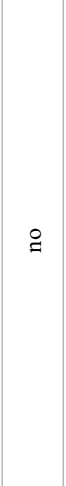 & $\Xi$ & 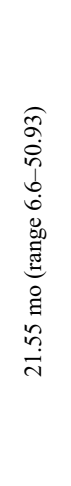 & 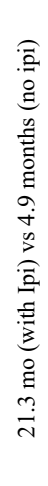 & $\Xi$ & $\vec{Z}$ & $\Xi$ & $\ddot{Z}$ & $\vec{z}$ & $\vec{Z}$ & $\begin{array}{l}\underset{0}{0} \\
\underset{0}{0} \\
\frac{0}{2} \\
m\end{array}$ & 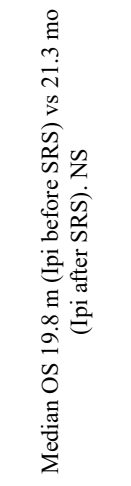 & $\vec{z}$ \\
\hline
\end{tabular}




\begin{tabular}{|c|c|c|c|c|c|c|c|c|c|c|c|c|c|c|c|c|c|c|c|c|}
\hline Patel 2015 & $\approx$ & $\Sigma$ & $\begin{array}{l}\overrightarrow{3} \\
+ \\
0 \\
\tilde{0} \\
\stackrel{\sim}{0}\end{array}$ & $\Xi$ & 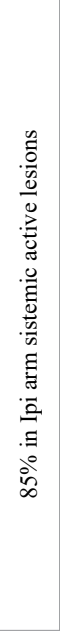 & 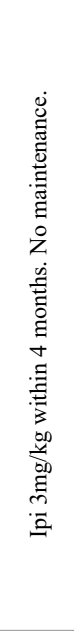 & 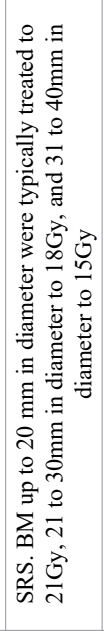 & 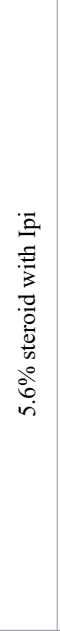 & 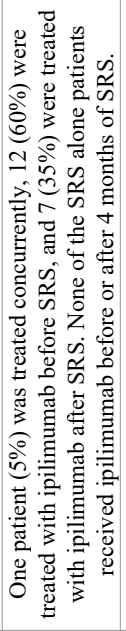 & 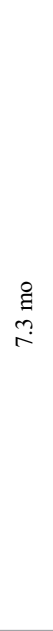 & $\Xi$ & $\Xi$ & 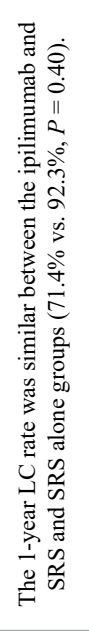 & $\Xi$ & $\Xi$ & $\vec{\Xi}$ & 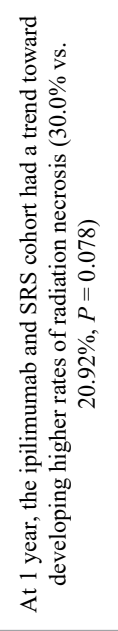 & 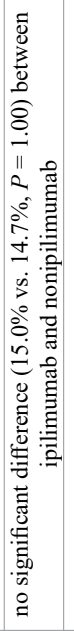 & 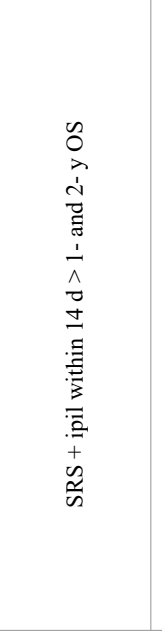 & $\Xi$ \\
\hline Gerber 2015 & $\simeq$ & $\Sigma$ & 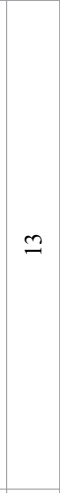 & $\begin{array}{l}\exists \\
\text { ڤें }\end{array}$ & $\Xi$ & 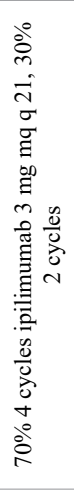 & 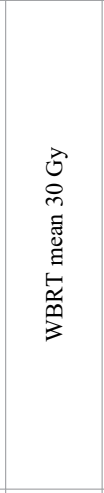 & 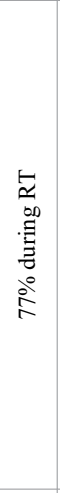 & 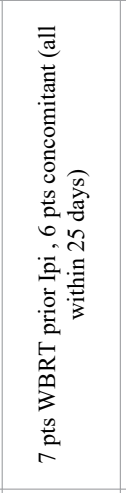 & 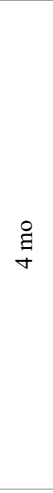 & $\stackrel{̊}{g}$ & $\Xi$ & $\Xi$ & $\begin{array}{l}\stackrel{0}{\circ} \\
\stackrel{0}{0} \\
\cong \\
\cong\end{array}$ & $\Xi$ & 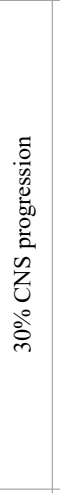 & 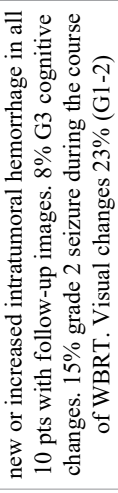 & (1) & $\Xi$ & 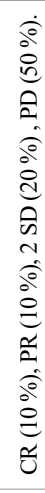 \\
\hline Silk 2013 & $\simeq$ & $\Sigma$ & 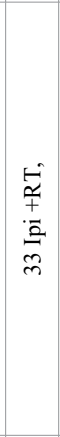 & $\begin{array}{l}\text { 岁 } \\
\text { ปें }\end{array}$ & $\Xi$ & 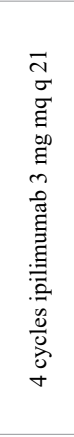 & 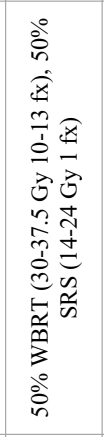 & $\Xi$ & 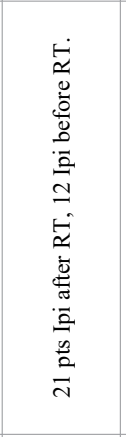 & $\Xi$ & 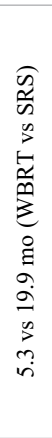 & $\begin{array}{l}\stackrel{̊}{g} \\
\hat{\tilde{E}}\end{array}$ & $\Xi$ & $\begin{array}{l}\tilde{6} \\
\text { ñ } \\
\cong\end{array}$ & $\Xi$ & $\Xi$ & 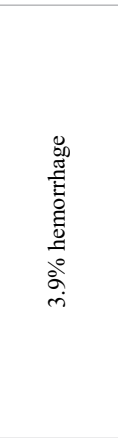 & 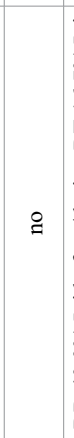 & 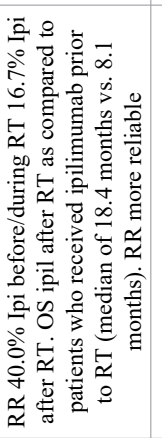 & $\Xi$ \\
\hline $\begin{array}{l}\text { Mathew } \\
2013\end{array}$ & $\simeq$ & $\Sigma$ & 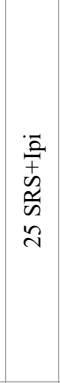 & $\Xi$ & ڤे & 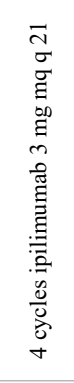 & $\begin{array}{l}\overrightarrow{3} \\
\stackrel{1}{1} \\
\tilde{\omega} \\
\tilde{\omega}\end{array}$ & 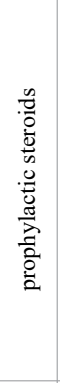 & 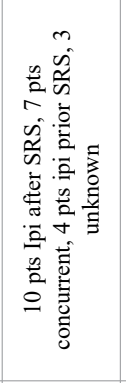 & 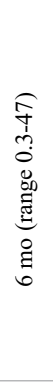 & 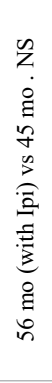 & $\Xi$ & 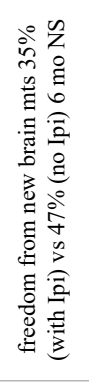 & 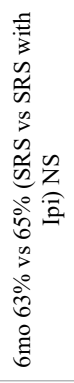 & $\Xi$ & $\Xi$ & 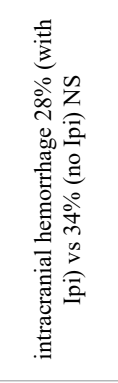 & 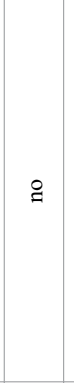 & 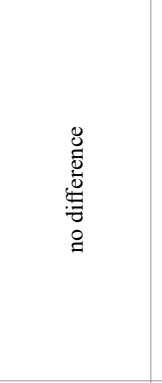 & $\Xi$ \\
\hline Tazi 2015 & $\approx$ & $\Sigma$ & 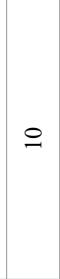 & $\rightleftarrows$ & $\Xi$ & 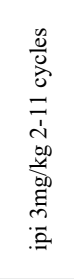 & $\tilde{\tilde{\omega}}$ & $\stackrel{\Xi}{ت}$ & 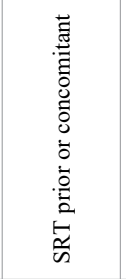 & $\Xi$ & 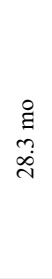 & $\Xi$ & $\Xi$ & $\Xi$ & 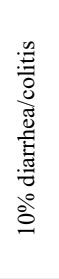 & $\Xi$ & @ & $\cong$ & $\rightleftarrows$ & $\Xi$ \\
\hline
\end{tabular}




\begin{tabular}{|c|c|c|c|c|c|c|c|c|c|c|c|c|c|c|c|c|c|c|c|c|}
\hline $\begin{array}{c}\text { Shoukat } \\
2013\end{array}$ & $\simeq$ & $\Sigma$ & $=$ & 商 & $\Xi$ & 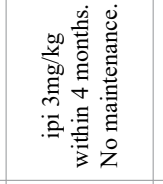 & $\tilde{\tilde{w}}$ & $\Xi$ & $\Xi$ & $\Xi$ & 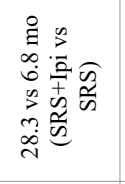 & $\Xi$ & $\Xi$ & $\Xi$ & 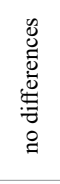 & $\Xi$ & 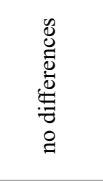 & $\Xi$ & $\stackrel{\Xi}{\Xi}$ & $\vec{g}$ \\
\hline Qian 2016 & $\simeq$ & $\Sigma$ & $\stackrel{n}{n}$ & $\Xi$ & $\frac{\circ}{\infty}$ & 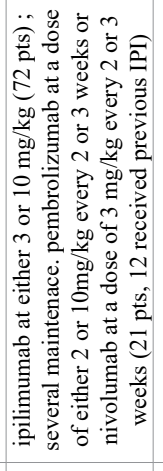 & 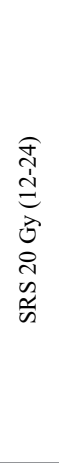 & $\Xi$ & 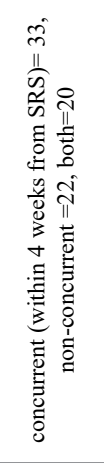 & 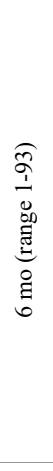 & $\begin{array}{l}\stackrel{0}{\Xi} \\
\omega \\
\infty \\
\infty\end{array}$ & $\Xi$ & $\Xi$ & $\Xi$ & $\Xi$ & 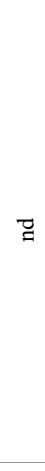 & $\Xi$ & $\stackrel{\circ}{\leftrightarrows}$ & 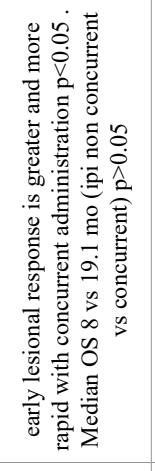 & $\Xi$ \\
\hline Jiang 2015 & $\simeq$ & $\Sigma$ & F & $\Xi$ & $\Xi$ & 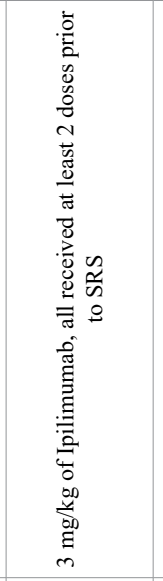 & $\begin{array}{l}\vec{b} \\
\text { ते } \\
\tilde{\tilde{n}} \\
\tilde{n}\end{array}$ & $\Xi$ & 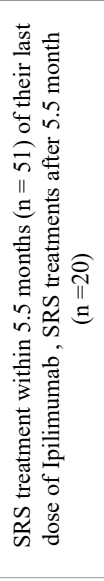 & $\Xi$ & 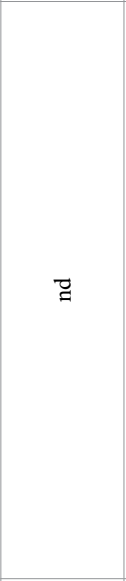 & $\Xi$ & $\Xi$ & 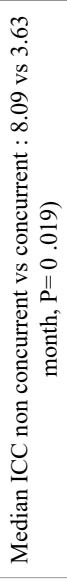 & $\Xi$ & $\Xi$ & 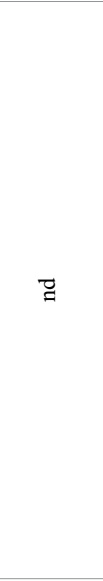 & $\Xi$ & 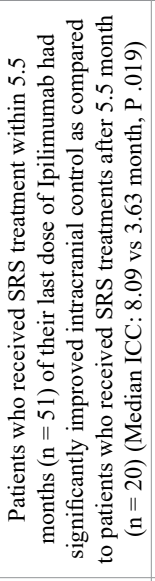 & $\Xi$ \\
\hline $\begin{array}{l}\text { Grimaldi } \\
2014\end{array}$ & $\approx$ & $\Sigma$ & $m$ & ॠ & $\Xi$ & 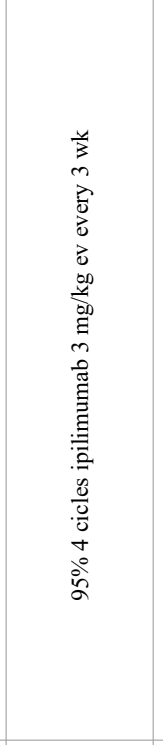 & 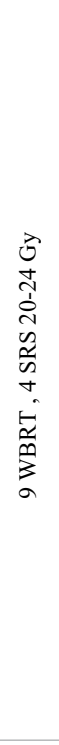 & $\stackrel{\Xi}{\Xi}$ & 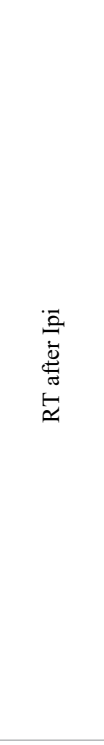 & $\stackrel{\circ}{\Xi}$ & 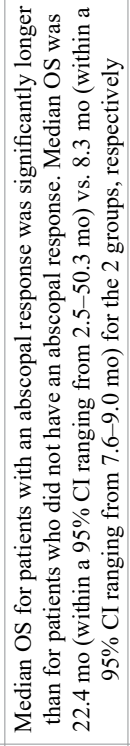 & $\begin{array}{l}\Xi \\
\Xi \\
\Xi\end{array}$ & $\Xi$ & $\Xi$ & $\Xi$ & $\Xi$ & $\Xi$ & $\Xi$ & $\Xi$ & 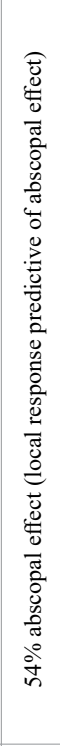 \\
\hline Khoja 2015 & $\simeq$ & $\Sigma$ & $\stackrel{ \pm}{m}$ & $\Xi$ & $\stackrel{\square}{z}$ & 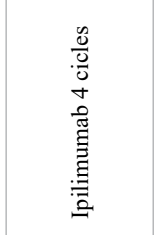 & 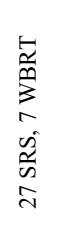 & $\stackrel{\square}{\nexists}$ & $\Xi$ & 曽 & \begin{tabular}{l}
$\stackrel{0}{g}$ \\
\multirow{b}{0}{}
\end{tabular} & 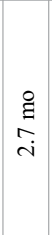 & $\Xi$ & $\Xi$ & $\Xi$ & $\begin{array}{l}\text { ¿े } \\
\stackrel{\circ}{=}\end{array}$ & 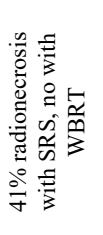 & ळे & $\ddot{z}$ & $\Xi$ \\
\hline
\end{tabular}




\begin{tabular}{|c|c|c|c|c|c|c|c|c|c|c|c|c|c|c|c|}
\hline $\begin{array}{l}\text { Ahmed } \\
2016\end{array}$ & $\approx$ & $\Sigma$ & $\AA$ & $\Xi$ & $\Xi$ & $\begin{array}{l}\text { 营 } \\
\text { 产 } \\
\text { 关 }\end{array}$ & $\begin{array}{l}\vec{w} \\
\tilde{w} \\
\tilde{n} \\
\tilde{n}\end{array}$ & $\Xi$ & & & $\stackrel{\varrho}{\cong}$ & & & $\begin{array}{l}\stackrel{\lambda}{\circ} \\
\stackrel{\infty}{\infty}\end{array}$ & 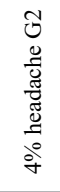 \\
\hline $\begin{array}{l}\text { Ahmed } \\
2016\end{array}$ & $\simeq$ & $\Sigma$ & $\stackrel{ \pm}{m}$ & $\Xi$ & $\Xi$ & 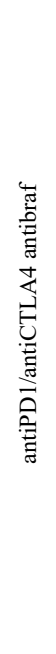 & 苂 & $\Xi$ & 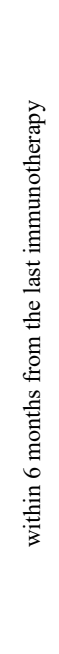 & 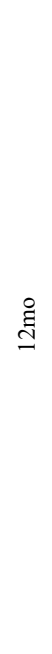 & 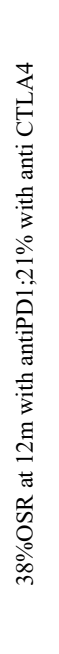 & $\Xi$ & 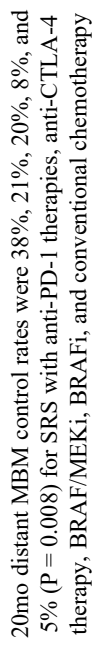 & & \\
\hline Ahmed KA & $\approx$ & $\Sigma$ & i & $\Xi$ & & $\begin{array}{l}\text { 言 } \\
\text { 言 }\end{array}$ & $\tilde{\tilde{\omega}}$ & $\hat{z}$ & 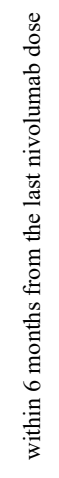 & 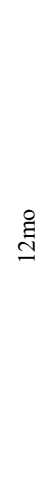 & $\begin{array}{l}\stackrel{\varrho}{g} \\
\stackrel{\infty}{=}\end{array}$ & $\Xi$ & 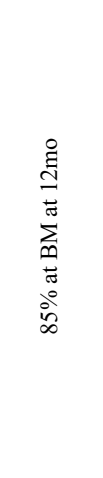 & 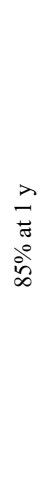 & \\
\hline
\end{tabular}

Abbreviations STUDY $\mathrm{R}=$ retrospective; $\mathrm{M}=$ melanoma; $\mathrm{MA}=$ multivariate analysis

is shown that 3 patients had brain metastases, and 44 pts underwent some kind of radiation therapy. No further information about outcome in patients with brain metastases or patient who had radiation therapy.

\section{Nivolumab}

Nivolumab is a fully human immunoglobulin (Ig) G4 monoclonal antibody directed against the negative immunoregulatory human cell surface receptor programmed death-1 (PD-1, PDL-1,) with immune checkpoint inhibitory and antineoplastic activities. It is approved for advanced malignant melanoma, urothelial cancer and squamous NSCLC

It is actually under investigation in melanoma symptomatic brain metastases (CA209-322).

The combination of Nivolumab and RT was reported by researchers of Moffitt Cancer Center, in a retrospective analyses on 26 patients with 73 brain metastases, RT was administered prior to, during, and after nivolumab in 33 lesions (45\%), 5 lesions (7\%) and 35 lesions (48 $\%)$, respectively. Local brain metastases control following RT were $91 \%$ after 6 months and $85 \%$ after 12 months. Median OS was 11.8 and 12.0 months, respectively, in patients receiving nivolumab for unresected disease. Median OS was not reached in patients treated in the resected setting [26].

In the study, all brain metastases were treated with stereotactic radiosurgery (SRS) in a single session except for 12, who were treated with fractionated stereotactic radiation therapy, nine of whom were in the postoperative setting. No other treatment-related neurologic toxicities or scalp reactions were reported. Grant et al presented at ASCO 2016 preliminary results of an open-label, multisite phase 2 study on pts with active asymptomatic melanoma BM with no prior local therapy to the brain. Treatment consists of 3 cohorts. (nivolumab only vs nivolumab combined with ipilimumab in asymptomatic pts cohort1 and 3; and symptomatic patients cohort 2). The primary endpoint is the best intracranial response $\geq \mathrm{wk} 12$. Secondary endpoints include best extracranial response, best overall response, intracranial PFS, extracranial PFS, overall PFS and overall survival, as well as safety and tolerability. The study will continue with an additional two cohorts of nivolumab combined with stereotactic radiosurgery $(\leq 4$ brain metastases) or whole brain radiotherapy ( $>5$ brain metastases) will be recruited. Clinical trial information: NCT02374242 [27]. Results on combination with RT are summarized in Table 1. 


\section{Results}

\section{Population}

Fifteen retrospective studies [23, 28-36] reported results of 594 melanoma patients with BM. Only 4 studies (Schoenfeld, Patel,Mathew, Qian) reported active systemic disease in the majority of patients (range 72\%-96\%). The described prognostic factors were KPS, DS-GPA, less frequently $\mathrm{LDH}$ serum levels and rarely B-RAF mutational state.

\section{Intervention}

Among the 594 with melanoma BM of the analyzed papers, 150 were treated with RT alone, 409 were treated with radiotherapy and Ipi, 26 with RT and nivolumab (Ahmed) and 12 with RT and pembrolizumab (3 previously treated also with ipilimumab) (Qian). The majority of patients received Ipi 4 cycles $3 \mathrm{mg} \mathrm{mq} \mathrm{q} \mathrm{21,} \mathrm{some} \mathrm{with} \mathrm{maintainance}$ every 3 months. Radiotherapy was in $84 \%$ a SRS treatment (mean 20 Gy in 1 fraction) and in 16\% WBRT (mean 30 Gy 10 fractions). The majority of the studies did not report about steroid use. When declared it was a short course profilactic use during RT [37-40].

Timing of the combination of radiotherapy and immunotherapy was described differently between studies where this aspect was considered. To define a concomitance between RT and immunotherapy some Authors have considered adequate that the treatments were not administered to more than 4 weeks, 3 months, 5.5 months of each other. In other cases, despite being administered with an interval less than a month the Authors distinguish patients in whom the immunotherapy was administered before, during or after the RT. Others described RT as administered before, during or after immunotherapy without a clear time interval $[41,42]$.

\section{Outcome}

The median of mean follow-up reported in the selected studies is 7.4 months (range 4-24).

The median survival shows great variability (range 4-56 months) as well as the survival at 1 year (range $15.4 \%-90 \%$ ).

Prognostic correlations on multivariate analysis reveal a better OS for patients with single brain metastasis and controlled systemic disease (Patel), higher msGPA scores, lower ECOG and LDH. Studies that compare patients treated with RT alone or with immunotherapy. find contradictory results on the impact of Ipi on OS with a significant better OS with the adjunct of ipilimumab in 2 studies (even if in the study by Silk and colleagues it was observed only in patients treated with SRS) whereas ipi has not a significant prognostic impact in the other two studies.

Influence of timing of the association between RT and immunotherapy on oncological end-points is analyzed in 8 papers (Schoenfeld, Kiess, Knisley, Patel, Silk, Mathew, Qian, Jiang). Only Mathew and colleagues find no correlation of the outcome with timing of administration of RT and Ipi. In the other 7 studies is reported a better OS with RT performed before or during immunotherapy and this correlation is statistically significant in 4 studies. Median PFS is reported only in 2 studies and is around 3 months.

Where reported, intracranial Response Rate is $56 \%$ and $85 \%$ at 1 year, or $65 \%$ at 6 months. Abscopal effect is reported in 4 studies with rates ranging from $20 \%$ to $68 \%$. A better "out-of-filed" response was associated with fraction size $</=3$ in one study, Ipi administered less than 3 months apart from SRS and local response. Incidence of systemic toxicity is reported only in 2 studies and referred to typical immune-related adverse events as diarrhea, colitis, pruritus, hepatitis. Discontinuation rate was analyzed in 3 studies with a range of 17$30 \%$, due to disease progression. CNS specific toxicity is reported in 9 studies. Radiation necrosis requiring surgery appears in 3\%-15\% of patients. Where feasible a comparison between patients treated with $\mathrm{RT}$ alone and RT+immune no differences of incidence of radiation necrosis emerge between groups. G3 CNS bleeding is reported in 15\% of patients, again without differences in patients treated with RT alone. Khoja et al. report an incidence of radiation necrosis in $41 \%$ of patients treated SRS compared with $0 \%$ in the WBRT group. Kiess et al show a higher CNS toxicity when RT is administered during Ipi (13\% G3 CNS bleeding vs $6 \%, 13 \% \mathrm{G} 3$ seizures vs $0 \%$ ).

\section{Ongoing trials}

The discovery that immunotherapy is safety in patients Table 2 summarizes the most impressive ongoing advanced clinical trials.

\section{Conclusion}

Immunotherapy is now a key player in Oncology. Brain metastasis are associated with poor prognosis and lack of effective systemic treatments. Data on combination of immune checkpoints inhibitors and Rt are heterogeneous because of retrospective series, different population and response evaluation. Timing of immunoradiotherapy combination differ in many studies, some trials report benefit with irradiation after the last dose of immunotherapy, other during the treatment.

Another limit of a systematic review on immunoradiotherapy of $\mathrm{BM}$ is the assessment of tumor response. As already reported several instruments and end points were used in the different studies. Another limitation is that in the majority of studies no endpoints other than survival is addressed. Results from ongoing trials on BM immunoRT will help to define the optimal association, schedule and the ideal setting of patients.

Table 2. Ongoing trials with rt and immunotherapy.

\begin{tabular}{|c|c|c|c|}
\hline Study & Setting & Drug & Expeted results \\
\hline CA209-322 & Mel symptomatic BM & $\begin{array}{c}\text { Nivo in symptomatic } \\
\text { BM }\end{array}$ & Jan April 2017 \\
\hline NCT02681549 & BM in NSCLC & $\begin{array}{l}\text { Pembro } \pm \text { Beva in } \\
\text { melanoma e NSCLC }\end{array}$ & May October 2019 \\
\hline NCT01727531 & $\mathrm{BM}^{*}$ & Chloroquine & Jan 2017 \\
\hline NCT02085070 & $\mathrm{BM}$ in NSCLC & Pembro and Beva & Dec 2018 \\
\hline NCT02097732 & Melanoma BM & $\mathrm{Ipi} \rightarrow \mathrm{SRT}$ & May 2017 \\
\hline $\begin{array}{l}\text { GEM study } \\
\text { GRAY-B* }\end{array}$ & Melanoma BM & Ipilimumab & Oct 2016 \\
\hline NCT02374242 & Melanoma BM & Nivo \pm Ipi & Dec 2017 \\
\hline NCT02808416 & $\mathrm{BM}^{*}$ & PERCELLVAC3 & Jun 2018 \\
\hline NCT02886585 & $\mathrm{BM}^{*}$ & Pembro+SRT & April2020 \\
\hline NCT02669914 & $\mathrm{BM}^{*}$ & durvalumab & Dec 2021 \\
\hline NCT02858869 & $\begin{array}{l}\text { Lung and melanoma } \\
\text { BM }\end{array}$ & Pembro+SRT & Oct 2019 \\
\hline NCT01703507 & Melanoma BM & Ipi+SRT & Oct 2019 \\
\hline NCT02696993 & Lung BM & $\begin{array}{c}\text { Nivo } \pm \text { Ipi+SRT/ } \\
\text { WBRT }\end{array}$ & Dec 2020 \\
\hline NCT02320058 & Melanoma BM & Nivo+Ipi $\square$ Nivo & Jun2018 \\
\hline
\end{tabular}

Abbreviations $\mathrm{BM}=$ brain metastases; $\mathrm{NSCLC}=$ non small cell lung cancer; SRT stereotactic RT; Nivo= nivolumab; Ipi= Ipilimumab;Pembro=pembrolizumab;"advanced solid tumours;AB.E abscopal effect. Early result presented at ESMO $20161 \mathrm{y}$ survival rate $31.4 \%$ 


\section{Evaluation}

Response to cancer therapy has been historically defined using RECIST criteria, however in the era of immunotherapy discordance in clinical and instrumental behaviour led to the identification of more suitable criteria. Using RECIST 1.1 in immunotherapy trials would lead to declaration of progressive disease (PD) too early, when the treatment effect is not yet fully evident. RECIST also neglects the importance of the 'flare effect' - pseudo-progression effect within the so-called flare time window.

Immune related Response Criteria (irRC) based on WHO criteria were published with an aim to provide better assessment of the effect of immunotherapeutic agents. Hodi et al demonstrated in Keynote 001 that conventional RECIST might underestimate the benefit of pembrolizumab in approximately $15 \%$ of patients.

Only in the last two three years patients with BM were enrolled in clinical trials. These studies assessed BM response using alternatively modified RECIST, RANO-BM (Response Assessment in NeuroOncology) criteria. and functional outcomes. For example, in recent times a number of trials have used time to neurologic progression or decline as primary endpoint. Focusing not only on survival could lead to differentiate between death resulting from systemic cancer progression or neurological decline from BM. Other outcomes include proportion of patients using steroids to control of cerebral edema for greater than $96 \mathrm{~h}$ and brain progression free survival. Recent trials investigated response using interval changes in dynamic MRI parameters such as perfusion, blood volume, vascular permeability and diffusion tensor imaging; moreover the change in $3 \mathrm{D}$ tumor volume is also under investigation.

A new instrumental evaluation includes FET PET (18F-fluoroethyl-tyrosine [FET]),that is actually compared to MRI brain scans to assess metabolic response of the BM to immune therapy. Several studies showed that FET-PET is very sensitive in the management of brain tumours. It is hypothesized FET-PET may overcome the difficult of MRI to evaluate lymphocytic infiltration.

\section{References}

1. Nayak L, Lee EQ, Wen PY (2012) Epidemiology of brain metastases. Curr Oncol Rep 14: 48-54. [Crossref]

2. Tsao MN, Lloyd N, Wong RK (2012) Whole brain radiotherapy for the treatment of newly diagnosed multiple brain metastases. Cochrane Database Syst Rev 4:CD003869

3. Ahluwalia MS, Vogelbaum MV, Chao ST, Mehta MM (2014) Brain metastasis and treatment. F1000Prime Rep 6: 114. [Crossref]

4. Venur VA, Peereboom DM, Ahluwalia MS (2015) Current medical treatment of glioblastoma. Cancer Treat Res 163: 103-115. [Crossref]

5. Nguyen DX, Bos PD, Massagué J (2009) Metastasis: from dissemination to organspecific colonization. Nat Rev Cancer 9: 274-284. [Crossref]

6. Muldoon LL, Soussain C, Jahnke K, Johanson C, Siegal T, et al. (2007) Chemotherapy delivery issues in central nervous system malignancy: a reality check. J Clin Oncol 25: 2295-2305. [Crossref]

7. Percy DB, Ribot EJ, Chen Y, McFadden C, Simedrea C, et al. (2011) In vivo characterization of changing blood-tumor barrier permeability in a mouse model of breast cancer metastasis: a complementary magnetic resonance imaging approach. Invest Radiol 46: 718-725.

8. Rudà R, Franchino F, Soffietti R (2016) Treatment of brain metastasis: current status and future directions. Curr Opin Oncol 28: 502-510. [Crossref]

9. Kibbi N, Kluger H (2016) The Treatment of Melanoma Brain Metastases. Curr Oncol Rep 18: 73. [Crossref]

10. Coutinho AE, Chapman KE (2011) The anti-inflammatory and immunosuppressive effects of glucocorticoids, recent developments and mechanistic insights. Mol Cell Endocrinol 335: 2-13. [Crossref]
11. Moher D, Shamseer L, Clarke M, Ghersi D, Liberati A, et al. (2015) Preferred reporting items for systematic review and meta-analysis protocols (PRISMA-P) 2015 statement. Syst Rev 4: 1. [Crossref]

12. Franceschini D, Franzese C, Navarria P, Ascolese AM, De Rose F, et al. (2016) Radiotherapy and immunotherapy: Can this combination change the prognosis of patients with melanoma brain metastases. Cancer Treat Rev 50: 1-8.

13. Davies MA (2012) Targeted therapy for brain metastases. Adv Pharmacol 65: 109-142. [Crossref]

14. Galea I, Felton LM, Waters S, van Rooijen N, Perry VH (2008) Newman TA. Immuneto-brain signalling: the role of cerebral CD163-positive macrophages. Neurosci Lett 448: 41-46.

15. Goldmann J, Kwidzinski E, Brandt C, Mahlo J, et al. (2006) Cells traffic from brain to cervical lymph nodes via the cribroid plate and the nasal mucosa. J Leukoc Biol 2006 80: 797-801.

16. Van Zwam M, Huizinga R, Melief MJ, Wierenga-Wolf AF, Van Meurs M, et al. (2009) Brain antigens in functionally distinct antigen-presenting cell populations in cervical lymph nodes in MS and EAE. J Mol Med 87: 273-86.

17. Ransohoff RM, Engelhardt B (2012) The anatomical and cellular basis of immune surveillance in the central nervous system. Nat Rev Immunol 12: 623-635. [Crossref]

18. Stahn C, Buttgereit F (2008) Genomic and nongenomic effects of glucocorticoids. Nat Clin Pract Rheumatol 4: 525-533. [Crossref]

19. Kadmiel M, Cidlowski JA (2013) Glucocorticoid receptor signaling in health and disease. Trends Pharmacol Sci 34: 518-530. [Crossref]

20. Zen M, Canova M, Campana C, Bettio S, Nalotto L, et al. (2011) The kaleidoscope of glucorticoid effects on immune system. Autoimmun Rev 10: 305-310. [Crossref]

21. Derer A, Frey B, Fietkau R, Gaipl US (2016) Immune-modulating properties of ionizing radiation: rationale for the treatment of cancer by combination radiotherapy and immune checkpoint inhibitors. Cancer Immunol Immunother 65: 779-786. [Crossref]

22. Goldberg SB, Gettinger SN, Mahajan A, Chiang AC, Herbst RS, et al. (2016) Pembrolizumab for patients with melanoma or non-small-cell lung cancer and untreated brain metastases: early analysis of a non-randomised, open-label, phase 2 trial. Lancet Oncol 17: 976-983. [Crossref]

23. Schoenfeld JD, Mahadevan A, Floyd SR, Dyer MA, Catalano PJ, et al. (2015) Ipilmumab and cranial radiation in metastatic melanoma patients: a case series and review. J Immunother Cancer 15: 3-50.

24. Saraceni C, Kincaid H, McIntosh AF, Nair S (2016) Synergy of immune checkpoint targeting and radiation when used sequentially for the treatment of melanoma brain metastases. J Clin Oncol 34.

25. Grenga I, Donahue RN, Lepone LM, Richards J, Schlom J (2016) A fully human IgG1 anti-PD-L1 MAb in an in vitro assay enhances antigen-specific T-cell responses. Clin Transl Immunology 5: e83.

26. Ahmed KA, Abuodeh YA, Echevarria MI, Arrington JA, Stallworth DG, et al. (2016) Clinical outcomes of melanoma brain metastases treated with stereotactic radiosurgery and anti-PD-1 therapy, anti-CTLA-4 therapy, BRAF/MEK inhibitors, BRAF inhibitor, or conventional chemotherapy. Ann Oncol 27: 2288-2294. [Crossref]

27. Long VG, Atkinson V, Menzies AM, Guminski AD, Sandhu SK, et al. (2016) A Randomized Phase 2 Study of Nivolumab and Nivolumab Combined With Ipilimumab in Patients (Pts) With Melanoma Brain Metastases: The Anti-PD1 Brain Collaboration. J Clin Oncol 34

28. Chandra RA, Wilhite TJ, Balboni TA, Alexander BM, Spektor A, et al. (2015) A systematic evaluation of abscopal responses following radiotherapy in patients with metastatic melanoma treated with ipilimumab. Oncoimmunology 4: e1046028.

29. Gerber NK, Young RJ, Barker CA, Wolchok JD, Chan TA, et al. (2015) Ipilimumab and whole brain radiation therapy for melanoma brain metastases. $J$ Neurooncol 121 : 159-165. [Crossref]

30. Kiess AP, Wolchok JD, Barker CA, Postow MA, Tabar V, et al. (2015) Stereotactic radiosurgery for melanoma brain metastases in patients receiving ipilimumab: safety profile and efficacy of combined treatment. J Radiat Oncol Biol Phys 92: 368-375.

31. Knisely JP, Yu JB, Flanigan J, Sznol M, Kluger HM, et al. (2012) Radiosurgery fo melanoma brain metastases in the ipilimumab era and the possibility of longer survival. J Neurosurg 117: 227-233. [Crossref]

32. Mathew M, Tam M, Ott PA, Pavlick AC, Rush SC, et al. (2013) A Ipilimumab in melanoma with limited brain metastases treated with stereotactic radiosurgery. Melanoma Res 23: 191-195. 
33. Patel KR, Lawson DH, Kudchadkar RR, Carthon BC, Oliver DE, et al. (2015) Two heads better than one? Ipilimumab immunotherapy and radiation therapy for melanoma brain metastases. Neuro Oncol 17: 1312-1321.

34. Silk AW, Bassetti MF, West BT, Tsien CI, Lao CD (2013) Ipilimumab and radiation therapy for melanoma brain metastases. Cancer Med 2: 899-906. [Crossref]

35. Tazi K, Hathaway A, Chiuzan C, Shirai K (2015) Survival of melanoma patients with brain metastases treated with ipilimumab and stereotactic radiosurgery Cancer Med 4: 1-6.

36. Shoukat SM, David M, Rizzo M, Lawson DH, Liu Y, et al. (2013) Outcome with Stereotactic Radiosurgery (SRS) and Ipilimumab (Ipi) for Malignant Melanoma Brain Metastases. J Clin Oncol 31: 30-32.

37. Schadendorf D, Hodi FS, Robert C, Weber JS, Margolin K, et al. (2015) Pooled Analysis of Long-Term Survival Data From Phase II and Phase III Trials of Ipilimumab in Unresectable or Metastatic Melanoma. J Clin Oncol 33: 1889-1894. [Crossref]
38. Wolchok JD, Hoos A, O’Day S, Weber JS, Hamid O, et al. (2009) Guidelines for the evaluation of immune therapy activity in solid tumors: immune-related response criteria.Clin Cancer Res 15: 7412-7420.

39. Garon EB, Rizvi NA, Hui R, Leighl N, Balmanoukian AS, et al. (2015) Pembrolizumab for the treatment of non-small-cell lung cancer. $N$ Engl J Med 372: 2018-2028. [Crossref]

40. Fehrenbacher L, Spira A, Ballinger M, Kowanetz M, Vansteenkiste J, et al. (2016) Poplar Study Group.Atezolizumab versus docetaxel for patients with previously treated non-small-cell lung cancer (POPLAR): a multicentre, open-label, phase 2 randomised controlled trial. Lancet 387: 1837-1846.

41. Phillips C, Jeffree R, Khasraw M (2017) Management of breast cancer brain metastases: A practical review. Breast 31: 90-98. [Crossref]

42. Vatner RE, Cooper BT, Vanpouille-Box C, Demaria S, Formenti SC (2014) Combinations of immunotherapy and radiation in cancer therapy. Front Oncol 4: 325. [Crossref]

Copyright: (2017 Nerina D. This is an open-access article distributed under the terms of the Creative Commons Attribution License, which permits unrestricted use, distribution, and reproduction in any medium, provided the original author and source are credited. 